\title{
Correspondence
}

\section{QT interval: predictor of the plasma and myocardial concentrations of amiodarone}

Sir,

The suggestion by Debbas et al (1984; 51: 316-20) that prolongation of the QT interval correlates with plasma and myocardial concentrations in patients receiving long term oral treatment may have important implications for patients treated with intravenous amiodarone. We have treated a selected group of patients with acute myocardial infarction with intravenous amiodarone as a bolus of $5 \mathrm{mg} / \mathrm{kg}$ followed by an infusion of $1000 \mathrm{mg}$ over 24 hours. QTc intervals increased during the infusion in 11 of 12 subjects. The mean increase at the end of the 24 hours was $11 \%$ when the mean plasma concentration of amiodarone was 2.05 (range $0.9-4.0) \mathrm{mg} / \mathrm{l}$ and of the desethyl metabolite 0.23 (range $0 \cdot 1-0.4$ ) $\mathrm{mg} / \mathrm{l}$.

We conclude that serum amiodarone concentra- tions rise rapidly after intravenous administration, and this is associated with a lengthening of the QT interval, which may indicate myocardial impregnation and an antiarrhythmic effect. The desethyl metabolite is found in low concentrations within this 24 hour period compared with the usual plasma concentrations achieved in patients receiving prolonged oral treatment and is unlikely to be a major factor in any acute drug effects.

\section{Allen K Brown,} Johanna C Wiersum, Valerie Anderson, Royal Lancaster Infirmary, Lancaster.

\section{Effect of metoprolol on chest pain in acute myocardial infarction}

Sir,

In their paper on the "Effect of metoprolol on chest pain in acute myocardial infarction" (1984;51:438-44) Herlitz et al found that metoprolol reduced pain in the early period after myocardial infarction and attribute this to (unknown) anti-ischaemic effects and lowered oxygen consumption induced by the drug. While the latter seems a reasonable assumption the "antiischaemic" action of metoprolol is not entirely clear. Our own experience with the same drug given orally to volunteers may offer an explanation. We found a decrease in plasma viscosity and an increase in red cell deformability after medication. Both variables might be of importance, by enhancing collateral blood flow in the marginal zone around the myocardial necrosis. ${ }^{1}$ Hence it might be worth while considering whether changes in flow are partly the reason for the clinical benefit of metoprolol in acute myocardial ischaemia.

E Ernst,

A Matrai,

Klinik für Physikalische,

Medizin der Universität München, Ziemssenstrasse 1 ,

8000 München 2,

West Germany.

\section{Reference}

1 Dormandy J, Ernst E, Matrai A, Flute PT. Hemorrheologic changes following acute myocardial infarction. Am Heart f 1982; 104: 1364-7. 